\title{
AN INTERFEROMETRIC ELECTRON RULER WITH A PICOMETER ACCURACY IN GAUAGING LATTICE DISPLACEMENT
}

\author{
Lijun Wu, Yimei Zhu,* and J. Tafto \\ Materials Science Division, Brookhaven National Laboratory, Upton, NY 11973
}

We report a novel technique to accurately measure interfacial lattice displacement by forming an electron probe close to a specimen in a manner similar to that originally proposed by Gabor to record a hologram. ${ }^{1}$ This method is based on the quantitative analysis of the interference pattern of shadow images in coherent electron diffraction. The approach is unique in that there are no adjustable microscope parameters, the contrast is strong even when the fault is viewed edge-on, and a large number of shadow images of the fault corresponding to different Bragg reflections can be studied simultaneously. Since it is an interferometric technique, the spatial resolution of the measurement is not limited by the wave length of the fast electrons. 1pm accuracy has been demonstrated in measuring the displacement associated stacking faults and grin boundaries in $\mathrm{Bi}$ based superconductors. It is, to our knowledge, the highest that has been ever achieved in measurements of displacement vectors. ${ }^{2}$

Fig. 1 is schematics of the experimental set-up. Unlike conventional CBED, we focus the beam above the sample and thus obtain shadow images of the illuminated area in many reflections. Because of the high coherence of the beam, when a planar fault is probed, we see strong intensity oscillation, or interference pattern, parallel to the fault. The spacing of the oscillation depends on the magnification of the shadow image, or the distance from the beam cross-over to the sample, and the contrast of the pattern, which strongly depends on the reflections, is generally strongest for g.r $=2 \mathrm{n}+1$ but weakest for $2 \mathrm{n}$ (where $\mathrm{g}$ is the reflection, $\mathrm{r}$ the displacement, and $\mathrm{n}$ is an integer). The sign of the displacement can be determined by the symmetry of the pattern, while the accuracy in determining the amplitude of the displacement is significantly improved by examining high-order reflections. Fig.2a is a real-space image with the cross-over above a sample of $\mathrm{Bi}_{2} \mathrm{Sr}_{2} \mathrm{CaCu}_{2} \mathrm{O}_{8}$. It exhibits Fresnel fringes at the rim of the illuminated area, as well as a (002) lattice image of the crystal with a stacking fault marked by arrows. The contrast from this planar fault can be more clearly seen in the shadow dark-field images obtained in diffraction mode under the condition indicated in Fig.1. Experimental and simulated shadow images are shown in Fig.2b-h and Fig.3. In the experimental pattern of Fig.2b, the first reflection on the 001 row which is nearly lacking contrast is the $0,0,10$, suggesting a displacement vector $\mathrm{R}$ close to $c / 10=0.3087 \mathrm{~nm}$ (fig. $2 \mathrm{~d}$ ). A displacement of $c / 5=0.6174 \mathrm{~nm}$ can be ruled out because of the additional extinction at the 115 reflection (fig.2c). For larger g-vectors the sensitivity to displacement increases. We notice from the experimental pattern in fig.4a that it is the 1,1,29, rather than the $0,0,30$ reflection, that is closest to featureless, and this corresponds to a displacement of $3 c / 29=0.319 \mathrm{~nm}$. By further simulations and comparisons with experiment and quantitative error analysis procedure using $\chi^{2}=\Sigma\left(I^{\exp }-I^{c a l}\right)^{2} / \Sigma\left(I^{\exp }\right)^{2}$ analysis with. We determined for the extrinsic planar fault, $R=0.320 \pm 0.002 \mathrm{~nm}$; and for the intrinsic fault, $R=-0.319 \pm 0.001 \mathrm{~nm}\left(\chi^{2}<0.06\right)$. Rigid body translations associated with the (001) twist boundaries in $\mathrm{Bi}_{2} \mathrm{Sr}_{2} \mathrm{CaCu}_{2} \mathrm{O}_{8}$ were also studies. An example is shown in Fig.4. A displacement of $\mathrm{R}=0.026 \mathrm{~nm}$ has been determined for the boundary.

References

1. D. Gabor, Nature (London) 161, 777 (1948). 
2. L. Wu, Y. Zhu and J. Tafto, Phys. Rev. Lett. 85, 5126 (2000).

3. This work was supported by Division of Materials Sciences, U.S. Department of Energy, under contract No. DE-AC02-98CH10886.
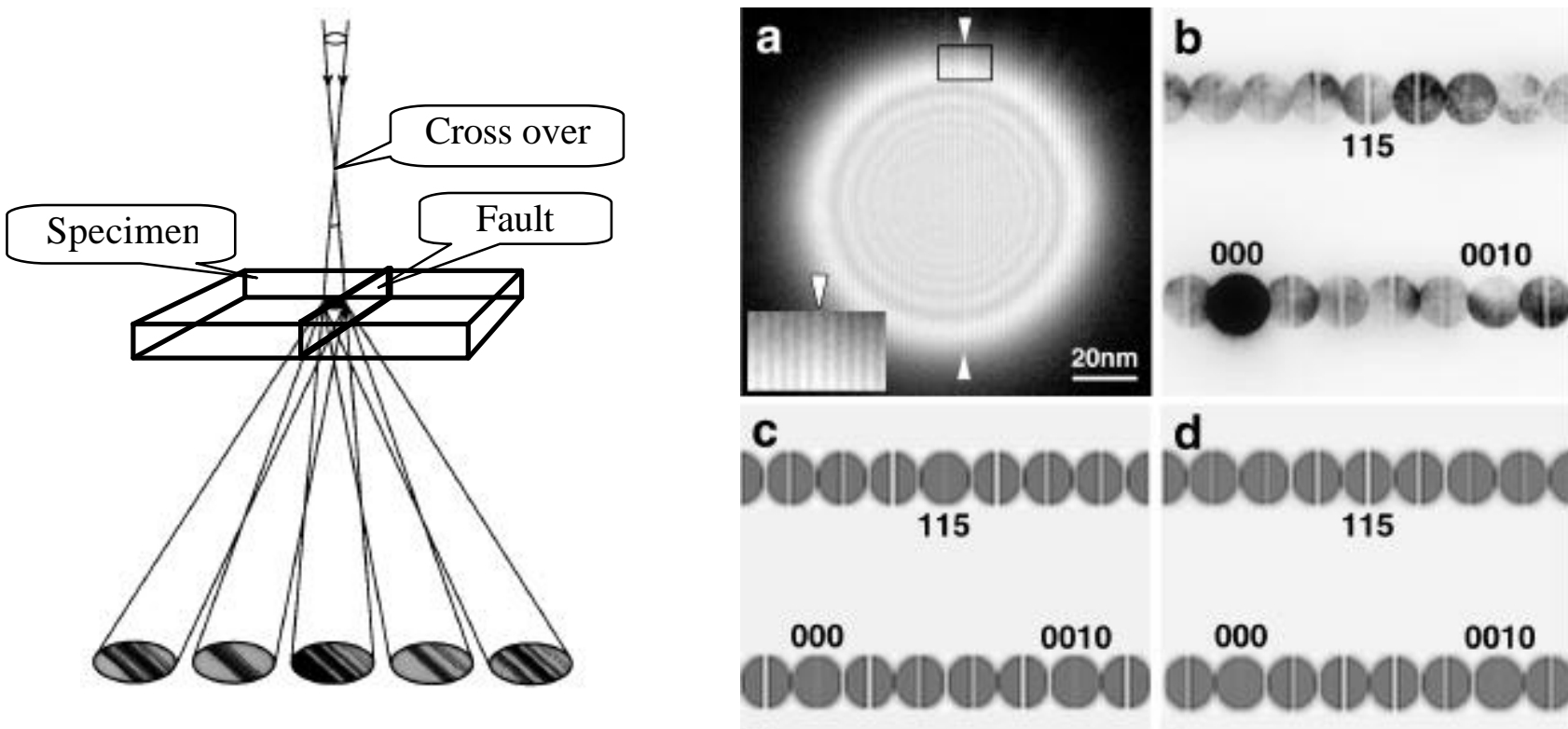

Fig.1 Experimental set-up showing the cross-over and shadow images in coherent electron diffraction.

a

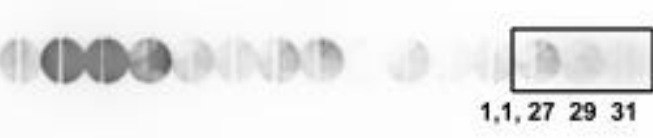

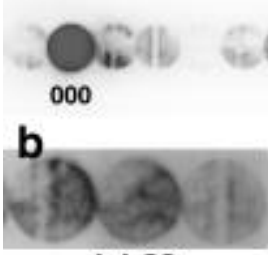

$1,1,29$

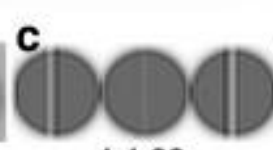

$1,1,29$

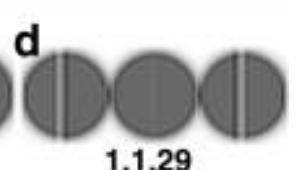

$1,1,29$
Fig.3(a) Shadow images of high-order reflections of the extrinsic fault in $\mathrm{Bi}_{2} \mathrm{Sr}_{2} \mathrm{CaCu}_{2} \mathrm{O}_{8}$ recorded with an imaging plate system. (b) Enlarged reflections of $1,1,27,1,1,29$, and 1,1,31. (c-d) Calculated reflections

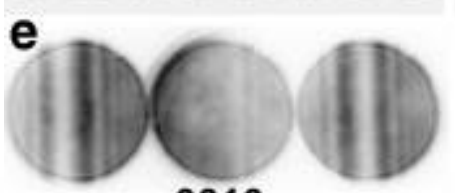

0010

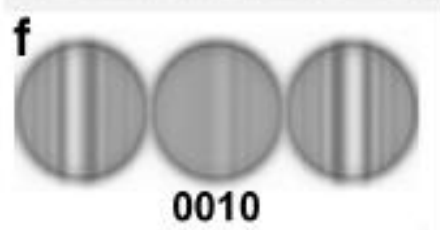

0010
Fig.2 An extrinsic stacking fault in $\mathrm{Bi}_{2} \mathrm{Sr}_{2} \mathrm{CaCu}_{2} \mathrm{O}_{8}$. (a) A real-space image. The inset is an enlargement of the boxed area, showing a wide lattice-fringe at the location of the fault denoted by an arrow. (b) Experimental diffraction pattern of the (1-10) ${ }^{*}$ zone recorded on a film negative. (c-d) Calculated patterns using the kinematical approach with $R=d_{005}=$ $0.6174 \mathrm{~nm}$ (c) and $R=d_{0010}=0.3087 \mathrm{~nm}(\mathrm{~d})$. (e) 008-0012 reflections recorded with a zero-loss energy-filtered CCD camera. (f) Calculated diffraction pattern with $R=0.322 \mathrm{~nm}$ giving a best fit to the 008-0012 reflections in (e). for $R=0.322 \mathrm{~nm}$ (c) and $R=0.320 \mathrm{~nm}(\mathrm{~d})$.

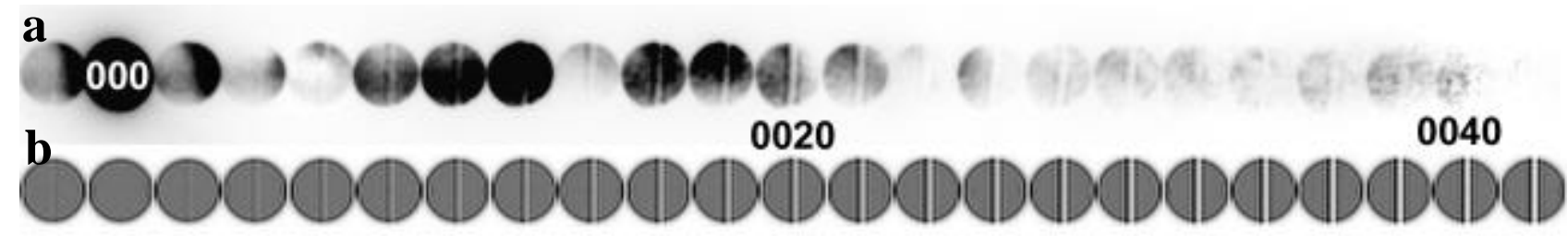

Fig.4 Experimental (a) and calculated (b) shadow-image diffraction patterns up to the 42th order of the (OOl) reflections of a $23.7^{\circ}(001)$ twist grain boundary in $\mathrm{Bi}_{2} \mathrm{Sr}_{2} \mathrm{CaCu}_{2} \mathrm{O}_{8}$. The best fit of the fault contrast to all reflections gives an interfacial displacement of $R=0.026 \mathrm{~nm}$. Note, the accuracy of the measurements also depends on the signal to noise ratio of the fault contrast, especially for high order reflections. For simplicity, in our calculations we did not scale the background intensity for each disks that is proportional to the structure factor squared of the individual reflections. 